\title{
Almost strictly sign regular matrices and Neville elimination with two-determinant pivoting
}

\author{
P. Alonso ${ }^{a}$, J.M. Peña ${ }^{\mathrm{b}}$, M.L. Serrano ${ }^{\mathrm{a}}$ \\ ${ }^{a}$ Departamento de Matemáticas, Universidad de Oviedo, Spain \\ ${ }^{b}$ Departamento de Matemática Aplicada, Universidad de Zaragoza, Spain
}

\begin{abstract}
In 2007 Cortés and Peña introduced a pivoting strategy for the Neville elimination of nonsingular sign regular matrices and called it two-determinant pivoting. Neville elimination has been very useful for obtaining theoretical and practical properties for totally positive (negative) matrices and other related types of matrices. A real matrix is said to be almost strictly sign regular if all its nontrivial minors of the same order have the same strict sign. In this paper, some nice properties related with the application of Neville elimination with two-determinant pivoting strategy to almost strictly sign regular matrices are presented.
\end{abstract}

Keywords: Almost strictly sign regular matrices, Neville elimination, two-determinant pivoting 2000 MSC: 65F05, 65F15, 65F35

\section{Introduction}

Sign Regular (SR) matrices are matrices whose minors of the same order have the same sign. These matrices arise naturally in many areas of mathematics, statistics, mechanics, computer-aided geometric design, economics, and others fields (see, for example, [4] and [9]). The interest of nonsingular SR matrices in many applications comes from their characterizations as variation-diminishing linear maps: the number of sign changes in the consecutive components of the image of a vector is bounded above by the number

Email addresses: palonso@uniovi.es (P. Alonso), jmpena@unizar.es (J.M. Peña), mlserrano@uniovi.es (M.L. Serrano) 
of sign changes in the consecutive components of the vector. If all minors of a matrix $A$ are nonnegative, then we say that $A$ is Totally Positive (TP).

TP matrices have been deeply studied in the literature (see [4], [9], [10] and [17]). A subclass of TP matrices with important applications is provided by Almost Strictly Totally Positive (ASTP) matrices (see [11] and [17]), which contain Hurwitz matrices and B-spline collocation matrices. Nonsingular ASTP matrices are TP matrices whose minors are positive if and only if all their diagonal entries are nonzero.

Almost Strictly Sign Regular (ASSR) matrices are introduced in [15], as those whose nontrivial minors of the same order have all the same strict sign. ASSR matrices form a subclass of SR matrices whose intersection with nonsingular TP matrices is the set of nonsingular ASTP matrices. Besides, ASSR matrices contain the class of Strictly Sign Regular (SSR) matrices, which are matrices whose minors of the same order have the same strict sign.

In [2] the authors present an algorithmic characterization of ASSR matrices using Neville Elimination (NE). A real matrix $A=\left(a_{i j}\right)_{1 \leq i, j, \leq n}$ is said to be Almost Strictly Totally Negative (ASTN) if it is ASSR with signature $\varepsilon=(-1,-1, \ldots,-1)$. So, in [3] an algorithmic characterization of nonsingular ASTN matrices is presented. All nontrivial minors of these matrices are strictly negative, which notably simplifies the characterization proposed in [2] for ASSR matrices. ASTN matrices contain all strictly totally negative matrices (which are matrices with all their minors negative) and are contained in the class of totally negative matrices (which are matrices with all their minors nonpositive). See [3, 5, 8, 9, 16] about these classes of matrices.

In this work we present some results about ASSR matrices when NE with two-determinant pivoting strategy is applied. NE is a classical method alternative to Gaussian elimination, and it plays an important role when dealing with SR matrices including totally positive and totally negative matrices. Roughly speaking, NE consists of making zeros in a column of a matrix by adding to each row an adequate multiple of the previous one, instead of using just a row with a fixed pivot as in Gaussian elimination. Other advantages of NE can be seen in $[1,12,13]$.

In the case of nonsingular TP matrices it can be applied without row exchanges (see [10]). However, this does not hold for nonsingular SR matrices, and so, the use of a pivoting strategy is necessary.

Taking into account the previous comments, Cortés and Peña introduce in [6] a pivoting strategy increasing $n$ operations to the cost of the NE of $n \times n$ nonsingular SR matrices. It is called two-determinant pivoting due to 
the special role played by some two by two determinants of some matrices appearing along the NE procedure.

In this paper we prove that the property of almost strict sign regularity is preserved under the NE with this pivoting strategy and that it presents optimal growth factor. Let us recall that the growth factor of a numerical algorithm is an indicator of its stability.

The paper is organized as follows: Section 2 is dedicated to preliminary concepts and notations. In Section 3 a short explanation about the Neville method is presented together with the two-determinant pivoting strategy, as well as some auxiliary results. Next section is dedicated to analyze the application of the NE with two-determinant pivoting to ASSR matrices. In Section 5 we prove that the growth factor is optimal when this pivoting strategy is applied to this kind of matrices and we analyze its application to ASTN matrices.

\section{Basic concepts and notations}

For $k, n \in \mathbb{N}$, with $1 \leq k \leq n, Q_{k, n}$ denotes the set of all increasing sequences of $k$ natural numbers not greater than $n$. For $\alpha=\left(\alpha_{1}, \ldots, \alpha_{k}\right)$, $\beta=\left(\beta_{1}, \ldots, \beta_{k}\right) \in Q_{k, n}$ and $A$ an $n \times n$ real matrix, we denote by $A[\alpha \mid \beta]$ the $k \times k$ submatrix of $A$ containing rows $\alpha_{1}, \ldots, \alpha_{k}$ and columns $\beta_{1}, \ldots, \beta_{k}$ of $A$. If $\alpha=\beta$, we denote by $A[\alpha]:=A[\alpha \mid \alpha]$ the corresponding principal submatrix. In addition, $Q_{k, n}^{0}$ denotes the set of increasing sequences of $k$ consecutive natural numbers not greater than $n$.

ASSR matrices present zero entries in certain positions, and can be classified in two classes that are defined below, type-I and type-II staircase.

A matrix $A=\left(a_{i j}\right)_{1 \leq i, j \leq n}$ is called type-I staircase if it satisfies simultaneously the following conditions

- $a_{11} \neq 0, a_{22} \neq 0, \ldots, a_{n n} \neq 0$;

- $a_{i j}=0, i>j \Rightarrow a_{k l}=0, \forall l \leq j, i \leq k$;

- $a_{i j}=0, i<j \Rightarrow a_{k l}=0, \forall k \leq i, j \leq l$.

From now on, we will be frequently using the backward identity matrix $n \times n, P_{n}$, whose element $(i, j)$ is defined as

$$
\left\{\begin{array}{cc}
1, & \text { if } i+j=n+1 \\
0, & \text { otherwise. }
\end{array}\right.
$$


So, $A$ is a type-II staircase matrix if it satisfies that $P_{n} A$ is a type-I staircase matrix.

To describe clearly the zero pattern of a nonsingular matrix $A$ type-I staircase (or type-II staircase, using the $n \times n$ backward identity matrix $P_{n}$ ) we must introduce some notations. For a matrix $A=\left(a_{i j}\right)_{1 \leq i, j \leq n}$ type-I staircase, we define

$$
i_{0}=1, \quad j_{0}=1
$$

and for $k=1, \ldots, l$ :

$$
\begin{gathered}
i_{k}=\max \left\{i / a_{i j_{k-1}} \neq 0\right\}+1(\leq n+1), \\
j_{k}=\max \left\{j \leq i_{k} / a_{i_{k} j}=0\right\}+1(\leq n+1),
\end{gathered}
$$

where $l$ is given in this recurrent definition by $j_{l}=n+1$.

Analogously we define

$$
\widehat{j}_{0}=1, \quad \widehat{i}_{0}=1,
$$

and for $k=1, \ldots, r$ :

$$
\begin{gathered}
\widehat{j}_{k}=\max \left\{j / a_{\hat{i}_{k-1} j} \neq 0\right\}+1(\leq n+1), \\
\widehat{i}_{k}=\max \left\{i \leq \widehat{j}_{k} / a_{\widehat{i j}_{k}}=0\right\}+1(\leq n+1),
\end{gathered}
$$

where $\widehat{i}_{r}=n+1$.

Finally, we denote by $I, J, \widehat{I}$ and $\widehat{J}$ the following sets of indices

$$
\begin{aligned}
I & =\left\{i_{0}, i_{1}, \ldots, i_{l}\right\}, & J & =\left\{j_{0}, j_{1}, \ldots, j_{l}\right\} \\
\widehat{I} & =\left\{\widehat{i}_{0}, \widehat{i}_{1}, \ldots, \hat{i}_{r}\right\}, & \widehat{J} & =\left\{\widehat{j}_{0}, \widehat{j}_{1}, \ldots, \widehat{j}_{r}\right\}
\end{aligned}
$$

thereby defining the zero pattern of the matrix $A$.

Next, we present some definitions and basic results.

Definition 1. For a real matrix $A=\left(a_{i j}\right)_{1 \leq i, j \leq n}$ type-I (type-II) staircase, a submatrix $A[\alpha \mid \beta]$, with $\alpha, \beta \in Q_{m, n}$, is nontrivial if all its main diagonal (secondary diagonal) entries are nonzero.

The minor associated to a nontrivial submatrix $(A[\alpha \mid \beta])$ is called a nontrivial minor $(\operatorname{det} A[\alpha \mid \beta])$. 
Definition 2. A vector $\varepsilon=\left(\varepsilon_{1}, \varepsilon_{2}, \ldots, \varepsilon_{n}\right) \in \mathbb{R}^{n}$ is a signature sequence, or simply, a signature, if $\left|\varepsilon_{i}\right|=1, \forall i \in \mathbb{N}, i \leq n$.

Definition 3. A real $n \times n$ matrix $A$ is said to be ASSR with signature $\varepsilon=$ $\left(\varepsilon_{1}, \varepsilon_{2}, \ldots, \varepsilon_{n}\right)$ if it is either type-I or type-II staircase and all its nontrivial minors $\operatorname{det} A[\alpha \mid \beta]$ satisfy that

$$
\varepsilon_{m} \operatorname{det} A[\alpha \mid \beta]>0, \quad \alpha, \beta \in Q_{m, n}, \quad m \leq n .
$$

Taking into account the previous definition, it is possible to show that any ASSR matrix is nonsingular. In fact, it is enough considering Eq. (7) with $\alpha=(1, \ldots, n)$ and $\beta=(1, \ldots, n)$. The next characterization for ASSR matrices follows from Theorem 10 of [15].

Theorem 1. Let $A$ be a real matrix $n \times n$ and $\varepsilon=\left(\varepsilon_{1}, \varepsilon_{2}, \ldots, \varepsilon_{n}\right)$ be a signature. Then $A$ is ASSR with signature $\varepsilon$ if and only if $A$ is a type-I or type-II staircase matrix and all its nontrivial minors with $\alpha, \beta \in Q_{m, n}^{0}$, $m \leq n$, satisfy

$$
\varepsilon_{m} \operatorname{det} A[\alpha \mid \beta]>0 .
$$

The next result (proved in [2]) establishes the relationship between the signatures of $A$ and $P_{n} A$.

Corollary 1. A matrix $A=\left(a_{i j}\right)_{1 \leq i, j \leq n}$ is $A S S R$ if and only if $P_{n} A$ is also ASSR. Furthermore, if the signature of $A$ is $\varepsilon=\left(\varepsilon_{1}, \varepsilon_{2}, \ldots, \varepsilon_{n}\right)$, then the

signature of $P_{n} A$ is $\varepsilon^{\prime}=\left(\varepsilon_{1}^{\prime}, \varepsilon_{2}^{\prime}, \ldots, \varepsilon_{n}^{\prime}\right)$, with $\varepsilon_{m}^{\prime}=(-1)^{\frac{m(m-1)}{2}} \varepsilon_{m}$, for all $m=1, \ldots, n$.

\section{NE, two-determinant pivoting and auxiliary results}

In this section we briefly present the NE and a row pivoting strategy associated to this method for nonsingular SR matrices. Besides, some results related to the application of this strategy are presented too.

$\mathrm{NE}$ is a very convenient procedure when working with ASSR matrices and other related types of matrices (see [2] and [10]). If $A$ is a nonsingular $n \times n$ matrix, NE consists of at most $n-1$ successive major steps, resulting in a sequence of matrices as follows:

$$
A=\widetilde{A}^{(1)} \rightarrow A^{(1)} \rightarrow \cdots \rightarrow \widetilde{A}^{(n)}=A^{(n)}=U
$$


where $U$ is an upper triangular matrix.

For each $t, 1 \leq t \leq n, A^{(t)}=\left(a_{i j}^{(t)}\right)_{1 \leq i, j \leq n}$ has zeros in the positions $a_{i j}^{(t)}$, for $1 \leq j \leq t, j \leq i \leq n$. Besides it holds that

$$
a_{i t}^{(t)}=0, i \geq t \Rightarrow a_{h t}^{(t)}=0, \forall h \geq i .
$$

The matrix $A^{(t)}$ is obtained from $\widetilde{A}^{(t)}$ reordering rows $t, t+1, \ldots, n$ according to a row pivoting strategy that satisfies (10).

To obtain $\widetilde{A}^{(t+1)}$ from $A^{(t)}$ we produce zeros in the column $t$ below the main diagonal by subtracting a multiple of the $i$ th row from the $(i+1)$ th, for $i=n-1, n-2, \ldots, t$, according to the following formula:

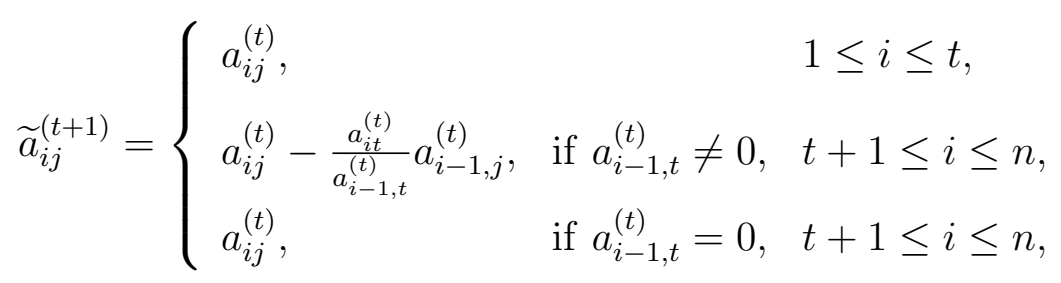

for all $j=1,2, \ldots, n$.

The element

$$
p_{i j}=a_{i j}^{(j)}, \quad 1 \leq j \leq i \leq n
$$

is called the $(i, j)$ pivot of $\mathrm{NE}$ of $A$ and the number

$$
m_{i j}=\left\{\begin{array}{cc}
\frac{a_{i j}^{(j)}}{a_{i-1, j}^{(j)}}\left(=\frac{p_{i j}}{p_{i-1, j}}\right), & \text { if } a_{i-1, j}^{(j)} \neq 0, \\
0, & \text { if } a_{i-1, j}^{(j)}=0,
\end{array}\right.
$$

the $(i, j)$ multiplier. Note that $m_{i j}=0$ if and only if $p_{i j}=0$ an, by (10),

$$
m_{i j}=0 \Longrightarrow m_{h j}=0, \forall h>i .
$$

In [6] a row pivoting strategy associated to NE for nonsingular SR matrices is introduced. It will be called two-determinant pivoting strategy. The criterion of the two-determinant pivoting strategy to obtain $A^{(t)}[t, \ldots, n]$ from a reordering of the rows of $\widetilde{A}^{(t)}[t, \ldots, n]$ is the following:

- If $\widetilde{a}_{t t}^{(t)}=0$ : then we reverse the ordering of the rows, that is, $A^{(t)}[t, \ldots, n]:=$ $P_{t} \widetilde{A}^{(t)}[t, \ldots, n]$. 
- If $\widetilde{a}_{n t}^{(t)}=0$ : then we do not perform rows exchanges, that is, $A^{(t)}:=\widetilde{A}^{(t)}$.

- If $\widetilde{a}_{t t}^{(t)} \neq 0$ and $\widetilde{a}_{n t}^{(t)} \neq 0$, then we compute the determinant $d_{1}=$ $\operatorname{det} \widetilde{A}^{(t)}[t, t+1]$.

- If $d_{1}>0$ then $A^{(t)}:=\widetilde{A}^{(t)}$.

- If $d_{1}<0$ then $A^{(t)}[t, \ldots, n]:=P_{t} \widetilde{A}^{(t)}[t, \ldots, n]$.

- If $d_{1}=0$ then compute the determinant $d_{2}=\operatorname{det} \widetilde{A}^{(t)}[n-1, n \mid t, t+$ $1]$.

* If $d_{2}>0$ then $A^{(t)}:=\widetilde{A}^{(t)}$.

* If $d_{2}<0$ then $A^{(t)}[t, \ldots, n]:=P_{t} \widetilde{A}^{(t)}[t, \ldots, n]$.

Applying the two-determinant pivoting strategy at step $t$ of NE requires the following conditions: at least one of the elements $\widetilde{a}_{t t}^{(t)}$ or $\widetilde{a}_{n t}^{(t)}$ is different from zero and, if both are nonzero, then at least one of the minors $d_{1}$ or $d_{2}$ is different from zero. This holds for nonsingular SR matrices, as shown in [6], and so for ASSR matrices. In fact, by Theorem 3.4 of [6], when $A$ is a nonsingular SR matrix and we use two-determinant pivoting, $\widetilde{A}^{(t)}[t, \ldots, n]$ is $\mathrm{SR}$, and nonsingular because $A$ is nonsingular. In addition, by Lemma 3.2 of [6], its first $\left(\widetilde{a}_{t t}^{(t)}\right)$ and last $\left(\widetilde{a}_{n t}^{(t)}\right)$ entries of the first column cannot be simultaneously zero and the sign $\epsilon_{2}\left(\widetilde{A}^{(t)}[t, \ldots, n]\right)$ determines which of them cannot be zero. Finally, Lemma 3.3 of [6] implies the aforementioned property on $d_{1}, d_{2}$.

Remark 1. The computational cost of the NE without row exchanges for an $n \times n$ matrix coincide with the cost of Gaussian elimination without row exchanges. So it has a cost of $\frac{4 n^{3}+3 n^{2}-7 n}{6} \simeq \frac{2 n^{3}}{3}$ flops (floating-point operations). Using the two-determinant pivoting strategy this cost is increased with at most $2 n-2$ subtractions and $4 n-4$ multiplications.

The following result proved in [6] will be used.

Lemma 1. Let $A=\left(a_{i j}\right)_{1 \leq i, j \leq n}$ be a nonsingular matrix. Let us assume that the first step of $N E$ of $A$ can be applied without rows exchanges. Let $k \in\{1, \ldots, n\}$ be such that $a_{i 1} \neq 0$ for all $i \leq k$ and $a_{i 1}=0$ for $i>k$. Then the resulting matrix $\widetilde{A}^{(2)}$ satisfies, for any $\alpha \in Q_{r, n}^{0}$ with $1 \leq r \leq n-1$, and $\beta \in Q_{r, n}$ with $2 \leq \alpha_{1}, \beta_{1} \leq n-r+1$,

$$
\operatorname{det} \widetilde{A}^{(2)}[\alpha \mid \beta]=\frac{\operatorname{det} A\left[\alpha_{1}-1, \alpha \mid 1, \beta\right]}{a_{\alpha_{1}-1,1}}, \quad \text { if } \quad k \geq \alpha_{1}-1,
$$


and

$$
\operatorname{det} \widetilde{A}^{(2)}[\alpha \mid \beta]=\operatorname{det} A[\alpha \mid \beta], \quad \text { if } \quad k \leq \alpha_{1}-1 .
$$

Moreover, if $\alpha_{1}-1=k$, we have

$$
\operatorname{det} A\left[\alpha_{1}-1, \alpha \mid 1, \beta\right]=a_{\alpha_{1}-1,1} \operatorname{det} A[\alpha \mid \beta] .
$$

Notice that if $A$ is an ASSR matrix, then $k=i_{1}$, where the index $i_{1}$ represents the row in which appears the first zero in the first column of $A$.

Remark 2. Let $A$ be an $n \times n$ ASSR matrix (and thus, nonsingular),

- If $\varepsilon_{2}(A)=1$, then the first step of $N E$ is performed without rows exchanges. In addition, $A=\widetilde{A}^{(1)}=A^{(1)}$, and $A^{(1)}$ is ASSR with $\varepsilon_{2}\left(A^{(1)}\right)=1$.

- If $\varepsilon_{2}(A)=-1$, then $A^{(1)}=P_{n} \widetilde{A}^{(1)}=P_{n} A$, and by Corollary $1 A^{(1)}$ is ASSR with $\varepsilon_{2}\left(A^{(1)}\right)=1$. Besides, the first step of $N E$ of this matrix can be performed without rows exchanges.

In any case, $A^{(1)}$ is ASSR with $\varepsilon_{2}\left(A^{(1)}\right)=1$, and by Lemma 7 of [15] it is type-I staircase.

\section{Preservation of ASSR matrices through NE two-determinant pivoting}

In this section we analyze the application of the NE with two-determinant pivoting strategy to ASSR matrices.

Proposition 1. Let $A=\left(a_{1 \leq i, j \leq n}\right)$ be an ASSR type-I staircase matrix, with zero pattern $J=\left\{j_{0}, j_{1}, \ldots, j_{l-1}, j_{l}\right\}$ and $l \geq 2$. Then, the NE with twodeterminant pivoting strategy does not involve row exchanges until the step $t=j_{l-1}$.

Proof. Index $j_{l-1}$ of the zero pattern is obtained as

$$
j_{l-1}=\max \left\{j \leq i_{l-1} / a_{i_{l-1} j}=0\right\}+1 .
$$

Then, $a_{i_{l-1}, j_{l-1}-1}=0$, and since $A$ is type-I staircase matrix, $\forall i \geq i_{l-1}$, and $\forall j \leq j_{l-1}-1, a_{i j}=0$. In particular, if $i=n \geq i_{l-1}$ we have that the $n$th row of $A$ does not change when performing NE with two-determinant pivoting strategy while the corresponding entry is zero. Thus, we do not perform row exchanges, that is, $A^{(t)}:=\widetilde{A}^{(t)}$ until the step $t=j_{l-1}$, and the result holds. 
The next result shows that almost strict sign regularity is inherited by all matrices $\widetilde{A}^{(t)}[t, \ldots, n]$ when we apply NE with two-determinant pivoting.

Theorem 2. Let $A=\left(a_{i j}\right)_{1 \leq i, j, \leq n}$ be an ASSR matrix, and let us apply $N E$ with two-determinant pivoting strategy. Then, for all $t \in\{1, \ldots, n\}$, all matrices $\widetilde{A}^{(t)}[t, \ldots, n]$ are $A S S R$ and $\varepsilon_{1}(A)=\varepsilon_{1}\left(\widetilde{A}^{(t)}\right)$.

Proof. We will prove the result by induction on $n$. For $n=1$ the result is satisfied. Let us assume that it holds for $n-1$ and let us prove it for $n$.

ASSR matrices form a subclass of SR matrices, and so we can apply Theorem 3.4 of [6]. This result allows us to assure that $\varepsilon_{1}\left(\widetilde{A}^{(t)}\right)=\varepsilon_{1}(A)$ and that $\widetilde{A}^{(t)}[t, \ldots, n]$ is an SR matrix for all $t=1, \ldots, n$. So, the proof would be completed if we show that $\widetilde{A}^{(t)}[t, \ldots, n]$ is ASSR.

First, and taking into account Theorem 10 of $[15]$, we prove that $\widetilde{A}^{(2)}[2, \ldots, n]$ is ASSR. For this purpose, we analyze the entries of the matrix for testing whether it is type-I or type-II staircase. Let us consider the index $i_{1}$ of the zero pattern of $A^{(1)}$. This index indicates the row in which appears the first zero in the first column of $A^{(1)}$. If the first column of $A^{(1)}$ does not contain zero elements, then $i_{1}=n+1$.

Next, we are going to analyze the entries of the matrix $\widetilde{A}^{(2)}$. The entries of $\widetilde{A}^{(2)}$ can be expressed, for all $j=1, \ldots, n$, as

- $\widetilde{a}_{1 j}^{(2)}=a_{1 j}^{(1)}$;

- If $1<i<i_{1}$, then

$$
\widetilde{a}_{i j}^{(2)}=a_{i j}^{(1)}-\frac{a_{i 1}^{(1)}}{a_{i-1,1}^{(1)}} a_{i-1, j}^{(1)}=\frac{1}{a_{i-1,1}^{(1)}} \operatorname{det}\left(\begin{array}{cc}
a_{i-1,1}^{(1)} & a_{i-1, j}^{(1)} \\
a_{i 1}^{(1)} & a_{i j}^{(1)}
\end{array}\right)
$$

- If $i_{1} \leq i \leq n$, then $\widetilde{a}_{i j}^{(2)}=a_{i j}^{(1)}=0$.

By Remark 2, the matrix $A^{(1)}$ is type-I staircase and ASSR. Besides, when $i<i_{1}$ we have that $a_{i-1,1}^{(1)} \neq 0$. Now, we prove that the matrix $\widetilde{A}^{(2)}[2, \ldots, n]$ has zeros in the same positions as $A^{(1)}[2, \ldots, n]$, which is type-I staircase.

Let $a_{i j}^{(1)}$ be an entry of $A^{(1)}[2, \ldots, n]$, with, $2 \leq i<i_{1}$ and $2 \leq j \leq n$, and suppose that $a_{i j}^{(1)}=0$. Two possible cases are considered:

- If $i \geq j$ then $a_{i k}^{(1)}=0$, for all $k=1, \ldots, i$. Thus, $a_{i 1}^{(1)}=a_{i-1, j}^{(1)}=0$ and, by (18), we have $\widetilde{a}_{i j}^{(2)}=0$. 
- If $i<j$, then $a_{k j}^{(1)}=0$, for all $k=1, \ldots, j-1$. Thereby, $a_{i-1, j}^{(1)}=a_{i j}^{(1)}=0$ and, by (18), we obtain $\widetilde{a}_{i j}^{(2)}=0$.

Suppose now that $a_{i j}^{(1)} \neq 0$. Then, as $i<i_{1}$ we have that $a_{i-1,1}^{(1)} \neq 0$, and so $\operatorname{det}\left(\begin{array}{cc}a_{i-1,1}^{(1)} & a_{i-1, j}^{(1)} \\ a_{i 1}^{(1)} & a_{i j}^{(1)}\end{array}\right)$ is a nontrivial minor of $A^{(1)}$. Since this matrix is ASSR, this minor is nonzero.

Therefore, the matrix $\widetilde{A}^{(2)}[2, \ldots, n]$ has the nonzero elements in the same positions as $A^{(1)}$. Thus, it is type-I staircase and Theorem 1 can be applied.

Next, we prove that nontrivial minors of same order of $\widetilde{A}^{(2)}[2, \ldots, n]$, with consecutive rows and columns, have the same sign, and so, by Theorem 1 , $\widetilde{A}^{(2)}[2, \ldots, n]$ is ASSR.

Let $\operatorname{det} \widetilde{A}^{(2)}[i, i+1, \ldots, i+r-1 \mid j, j+1, \ldots, j+r-1]$ be an nontrivial minor of $\widetilde{A}^{(2)}[2, \ldots, n]$. So, $\widetilde{a}_{i j}^{(2)} \cdots \widetilde{a}_{i+r-1, j+r-1}^{(2)} \neq 0$ with $2 \leq i, j \leq n-r+1$. Taking into account that, considering $\alpha=(i, \ldots, i+r-1), \beta=(j, \ldots, j+r-1)$ and $k=i_{1}$, the first step of NE with two-determinant pivoting strategy can be performed without row exchanges, the hypotheses of Lemma 1 are satisfied. So, now we analyze the sign of the nontrivial minor of order $r$, according to the value of $i$.

First, notice that, if $A$ is an ASSR matrix, then $A^{(1)}$ is also an ASSR matrix. If it is not necessary to perform rows exchanges, then $A=A^{(1)}$, and otherwise, considering $A^{(1)}=P_{n} A$, by Corollary 1 , also $A^{(1)}$ is ASSR.

- If $i_{1} \geq i-1$, by (15)

$$
\operatorname{det} \widetilde{A}^{(2)}[\alpha \mid \beta]=\frac{\operatorname{det} A^{(1)}\left[\alpha_{1}-1, \alpha \mid 1, \beta\right]}{a_{\alpha_{1}-1,1}^{(1)}},
$$

and the sign of this minor is the same that $\varepsilon_{r+1}\left(A^{(1)}\right) \varepsilon_{1}\left(A^{(1)}\right)$.

- If $i_{1} \leq i-1$, by (16)

$$
\operatorname{det} \widetilde{A}^{(2)}[\alpha \mid \beta]=\operatorname{det} A^{(1)}[\alpha \mid \beta]
$$

whose sign is $\varepsilon_{r}\left(A^{(1)}\right)$.

Notice that if $A^{(1)}$ is ASSR, then its zero pattern is unique, and so the value $i_{1}$ is fixed. 
In the case that $i_{1}=n$ or $i_{1}=n+1$, the condition $i_{1}>i-1$ is always satisfied. Besides, if $a_{n-1,1}^{(1)} \neq 0$, then the sign of the minor is always $\varepsilon_{1}\left(A^{(1)}\right) \varepsilon_{r+1}\left(A^{(1)}\right)$.

Suppose now that $i_{1}<n$. Taking $i=i_{1}+1$, (19) and (20) are satisfied, and thus $\varepsilon_{r}\left(A^{(1)}\right)=\varepsilon_{1}\left(A^{(1)}\right) \varepsilon_{r+1}\left(A^{(1)}\right)$. In any case, when $i_{1}<n$ all minors of order $r$ have the same sign.

Thus, for any value of $i_{1}$, nontrivial minors of the same order have the same sign, and by Theorem 1 the matrix $\widetilde{A}^{(2)}[2, \ldots, n]$ is ASSR.

It has been proved that, given a $n \times n$ ASSR matrix, the $(n-1) \times(n-1)$ matrix obtained after performing a step of $\mathrm{NE}$ with two-determinant pivoting strategy, $\widetilde{A}^{(2)}[2, \ldots, n]$, is ASSR with $\varepsilon_{1}\left(\widetilde{A}^{(2)}\right)=\varepsilon_{1}(A)$. By the induction hypothesis, for all $t=1, \ldots, n-1$ the matrices $\widetilde{A}^{(t+1)}[t+1, \ldots, n]$ are ASSR, and the result follows.

Remark 3. In the proof of Theorem 2 it has been shown that $A^{(1)}[2, \ldots, n]$ and $\widetilde{A}^{(2)}[2, \ldots, n]$ have the zero entries exactly in the same positions. Since the first row is unchanged, we can state that $\widetilde{A}^{(2)}$ has zeros placed in the same positions as $A^{(1)}$, except those arising from the process of $N E$.

The following example illustrates the application of NE with two-determinant pivoting strategy to an ASSR matrix.

Example 1. Let $A$ be an ASSR matrix with signature $\varepsilon=(-1,1,-1,1,-1,-1)$ :

$$
A=\left(\begin{array}{cc|cccc}
-1 & -2 & 0 & 0 & 0 & 0 \\
-2 & -6 & -6 & -8 & 0 & 0 \\
\cline { 1 - 4 } 0 & -6 & -21 & -30 & -9 & 0 \\
0 & -8 & -30 & -48 & -42 & -28 \\
0 & 0 & -9 & -42 & -172 & -176 \\
0 & 0 & 0 & -28 & -176 & -259
\end{array}\right)
$$

Applying the NE with two-determinant pivoting strategy the following sequence of matrices is obtained:

$$
\begin{gathered}
A=\widetilde{A}^{(1)}=A^{(1)} \rightarrow \widetilde{A}^{(2)}=A^{(2)} \rightarrow \widetilde{A}^{(3)}=A^{(3)} \rightarrow \\
\widetilde{A}^{(4)}=A^{(4)} \rightarrow \widetilde{A}^{(5)} \rightarrow A^{(5)} \rightarrow \widetilde{A}^{(6)}=A^{(6)}=U
\end{gathered}
$$

where $U$ is an upper triangular matrix. 
When $t=2$, the matrix calculated is

$$
\widetilde{A}^{(2)}[2,3,4,5,6]=\left(\begin{array}{ccccc}
-2 & -6 & -8 & 0 & 0 \\
-6 & -21 & -30 & -9 & 0 \\
-8 & -30 & -48 & -42 & -28 \\
0 & -9 & -42 & -172 & -176 \\
0 & 0 & -28 & -176 & -259
\end{array}\right),
$$

with

$$
\varepsilon\left(\widetilde{A}^{(2)}[2,3,4,5,6]\right)=(-1,1,-1,1,1) .
$$

In the case $t=3$, we have

$$
\widetilde{A}^{(3)}[3,4,5,6]=\left(\begin{array}{cccc}
-3 & -6 & -9 & 0 \\
-2 & -8 & -30 & -28 \\
-9 & -42 & -172 & -176 \\
0 & -28 & -176 & -259
\end{array}\right), \varepsilon\left(\widetilde{A}^{(3)}[3,4,5,6]\right)=(-1,1,-1,-1),
$$

and when $t=4$, then

$$
\widetilde{A}^{(4)}[4,5,6]=\left(\begin{array}{ccc}
-4 & -24 & -28 \\
-6 & -37 & -50 \\
-28 & -176 & -259
\end{array}\right), \varepsilon\left(\widetilde{A}^{(4)}[4,5,6]\right)=(-1,1,1) .
$$

The two last steps $(t=5,6)$ enable us to obtain

$$
\begin{aligned}
\widetilde{A}^{(5)}[5,6]= & \left(\begin{array}{cc}
-1.0000 & -8.0000 \\
-3.3333 & -25.6667
\end{array}\right), \varepsilon\left(\widetilde{A}^{(5)}[5,6]\right)=(-1,-1), \\
& \widetilde{A}^{(5)}[6]=(-0.3000), \varepsilon\left(\widetilde{A}^{(6)}[6]\right)=(-1) .
\end{aligned}
$$

The previous results allows us to conclude that the matrices $\widetilde{A}^{(t)}[t, \ldots, 6]$ obtained are $A S S R$, and besides $\varepsilon_{1}(A)=\varepsilon_{1}\left(\widetilde{A}^{(t)}[t, \ldots, 6]\right)=-1$, for all $t \in$ $\{1, \ldots, 6\}$.

Finally, it is also interesting to observe that the sets of indices $I$ and $J$ associated to $A$ are

$$
\begin{aligned}
& I=\left\{i_{0}=1, i_{1}=3, i_{2}=5, i_{3}=6, i_{4}=7\right\}, \\
& J=\left\{j_{0}=1, j_{1}=2, j_{2}=3, j_{3}=4, j_{4}=7\right\},
\end{aligned}
$$

and that the NE with two-determinant pivoting strategy does not involve rows exchanges until the step $t=j_{3}=4$. 


\section{Optimal growth factor and ASTN matrices}

The growth factor is an indicator of the numerical stability of a numerical algorithm (cf. [14]), measuring the size of intermediate and final quantities relative to initial data. In this section we analyze the growth factor introduced by Wilkinson.

Let $A$ be an $n \times n$ matrix. The growth factor associated to NE with two-determinant pivoting is given by

$$
\rho(A)=\frac{\max _{i, j, t}\left|\widetilde{a}_{i j}^{(t)}\right|}{\max _{i, j}\left|a_{i j}\right|}
$$

where $\widetilde{A}^{(t)}=\left(\widetilde{a}_{i j}^{(t)}\right)_{1 \leq i, j \leq n}$ are the intermediate matrices of the elimination process as in (9).

In [7] the authors prove that when the matrix is SSR the growth factor is 1 . The following result shows that this property is also satisfied by ASSR matrices.

Theorem 3. Let $A=\left(a_{i j}\right)_{1 \leq i, j \leq n}$ be an $n \times n$ ASSR matrix. Then the growth factor (22) corresponding to NE with two-determinant pivoting is optimal:

$$
\rho(A)=1 \text {. }
$$

Proof. To prove the result it is sufficient to see that

$$
\left|a_{i j}^{(t+1)}\right| \leq\left|a_{i j}^{(t)}\right|, \quad t+1 \leq i, j \leq n, \quad 1 \leq t \leq n-1
$$

By Corollary 1, $\varepsilon_{1}\left(\widetilde{A}^{(t)}\right)=\varepsilon_{1}\left(A^{(t)}\right)$. Besides, Theorem 2 allows us to ensure that $\varepsilon_{1}(A)=\varepsilon_{1}\left(\widetilde{A}^{(t)}\right)$. Thus

$$
\operatorname{sign}\left(a_{i j}^{(t)}\right)=\operatorname{sign}\left(\frac{a_{i t}^{(t)}}{a_{i-1, t}^{(t)}} a_{i-1, j}^{(t)}\right) .
$$

Besides, taking into account (11), we have

$$
\operatorname{sign}\left(\widetilde{a}_{i j}^{(t+1)}\right)=\operatorname{sign}\left(a_{i j}^{(t)}\right),
$$

and the result follows. 
Next, an example to show the stability of the NE with two-determinant pivoting strategy is presented.

Example 2. The systems used in the test are of the form $A x=b$, where the matrix of coefficients $A$ is the matrix of Example 1. The solution vector $x$ has been chosen as a real random vector from the uniform distribution on the intervals $[0,1],[-1,1],[0,255],[1,10]$ and $[0,1000]$. Then $b$ was computed in a straightforward way as $b=A x$.

In the numerical experiments we have computed the Wilkinson growth factor (22), the componentwise backward error (see [14])

$$
\omega_{|A|,|b|}(\hat{x})=\max _{i} \frac{\left|r_{i}\right|}{(|A||\hat{x}|+|b|)_{i}}
$$

where $\hat{x}$ denote the computed solution and $r=b-A \hat{x}$, and the componentwise relative error

$$
\max _{i} \frac{\left|x_{i}-\hat{x}_{i}\right|}{\left|x_{i}\right|} .
$$

As for Wilkinson growth factor, the obtained value is equal to 1, as shown in Theorem 3.

Table 1 permits us to observe, regarding componentwise backward error, that it is small for all cases (approximately $10^{-16}$ or $10^{-17}$ ), while that the componentwise relative error is of order of $10^{-11}$ to $10^{-13}$. Taking into account these results we can conclude that NE provides satisfactory results when two-determinant pivoting strategy is applied.

\begin{tabular}{|c|c|c|}
\hline Int. Sol. & backward error & relative error \\
\hline \hline$[0,1]$ & $1.1405 \mathrm{e}-16$ & $1.7297 \mathrm{e}-11$ \\
{$[-1,1]$} & $7.0239 \mathrm{e}-17$ & $3.0155 \mathrm{e}-13$ \\
{$[0,255]$} & $1.1078 \mathrm{e}-16$ & $7.5848 \mathrm{e}-12$ \\
{$[1,10]$} & $9.9610 \mathrm{e}-17$ & $5.4432 \mathrm{e}-11$ \\
{$[0,1000]$} & $1.1625 \mathrm{e}-16$ & $5.1201 \mathrm{e}-12$ \\
\hline
\end{tabular}

Table 1: Numerical test: Example 2

The following example shows the performance of growth factor when the pivoting strategy is not considered. 
Example 3. Let us consider an ASSR matrix with signature $(-1,-1,1)$ :

$$
A=\left(\begin{array}{ccc}
-10^{-4} & -1 & -1 \\
-1 & -3 & -1 \\
-1 & -1 & 0
\end{array}\right)
$$

If we carry out NE with two-determinant pivoting strategy, the obtained growth factor is 1 . Nevertheless, if the method is carried out without pivoting, then $\rho(A)=3333$ and in this case the stability of the strategy is not assured.

In the same way as in the previous example, Table 2 allows us to observe that the componentwise backward error is approximately $10^{-16}$ or $10^{-17}$, and the componentwise relative error is of order of $10^{-17}$.

\begin{tabular}{|c|c|c|}
\hline Int. Sol. & backward error & relative error \\
\hline \hline$[0,1]$ & $3.6601 \mathrm{e}-16$ & $6.0503 \mathrm{e}-17$ \\
{$[-1,1]$} & $3.4479 \mathrm{e}-17$ & $4.0881 \mathrm{e}-17$ \\
{$[0,255]$} & $6.2783 \mathrm{e}-17$ & $9.6142 \mathrm{e}-17$ \\
{$[1,10]$} & $8.6397 \mathrm{e}-17$ & $9.2830 \mathrm{e}-17$ \\
{$[0,1000]$} & $6.8759 \mathrm{e}-17$ & $6.2100 \mathrm{e}-17$ \\
\hline
\end{tabular}

Table 2: Numerical test: Example 3

In the particular case when we are working with ASTN matrices, the following result shows that only two row exchanges are necessary along the process.

Theorem 4. Let $A=\left(a_{i j}\right)_{1 \leq i, j \leq n}$ be an ASTN matrix. If NE with twodeterminant pivoting strategy is applied to $A$, then it is only necessary to perform row exchanges for $t=1$ and $t=2$.

Proof. An ASTN matrix is an ASSR matrix with signature $\varepsilon=(-1,-1, \ldots,-1)$. Moreover, by Theorem 2.1 of [16], we can state that these matrices can only have zero entries in positions $(1,1)$ and $(n, n)$.

When we apply NE with two-determinant pivoting to $A$ and $t=1$, then $\widetilde{A}^{(1)}=A$. If $\widetilde{a}_{11}=a_{11}=0$ then we reverse the ordering of the rows, that is, $A^{(1)}=P_{n} \widetilde{A}^{(1)}=P_{n} A$. If $\widetilde{a}_{11}=a_{11} \neq 0$, and considering that the sign of $d_{1}=\operatorname{det} A[1,2]$ is equal that to the sign of $\varepsilon_{2}=-1$, then also $A^{(1)}=P_{n} A$.

Since $A$ is an ASTN matrix, and so ASSR, by Corollary 1 the ma$\operatorname{trix} A^{(1)}$ is also ASSR with signature $\varepsilon_{m}\left(A^{(1)}\right)=\varepsilon_{m}\left(\widetilde{A}^{(1)}\right)(-1)^{m(m-1) / 2}=$ $(-1)(-1)^{m(m-1) / 2}$, for $m=1, \ldots, n$. 
At the next step of $\mathrm{NE}(t=2)$, Remark 3 enables us to assure that $\widetilde{a}_{n 2}^{(2)} \neq 0$. Besides, by Theorem 2 the matrix $\widetilde{A}^{(2)}[2, \ldots, n]$ is ASSR and the sign of $d_{1}=\widetilde{A}^{(2)}[2,3]$ is $\varepsilon_{2}\left(\widetilde{A}^{(2)}\right)$. Using (19) for $i=2$ and since $i_{1} \geq n$, we have that $i_{1} \geq i-1$, and thus $\varepsilon_{2}\left(\widetilde{A}^{(2)}\right)=\varepsilon_{3}\left(A^{(1)}\right) \varepsilon_{1}\left(A^{(1)}\right)$. Since $\varepsilon_{3}\left(A^{(1)}\right)=$ $(-1)(-1)^{3 \times 2 / 2}=1$ and $\varepsilon_{1}\left(A^{(1)}\right)=-1$, then $\varepsilon_{2}\left(\widetilde{A}^{(2)}\right)=-1$, and so $d_{1}<0$. Thus $A^{(2)}[2, \ldots, n]=P_{n-1} \widetilde{A}^{(2)}[2, \ldots, n]$.

When $t=3$, the matrix $A^{(2)}[2, \ldots, n]$ is ASSR and $\varepsilon_{m}\left(A^{(2)}[2, \ldots, n]\right)=$ $\varepsilon_{m}\left(\widetilde{A}^{(2)}[2, \ldots, n]\right)(-1)^{m(m-1) / 2}$. Therefore, as in the previous case, and using (19), $\varepsilon_{2}\left(\widetilde{A}^{(3)}[3, \ldots, n]\right)=\varepsilon_{3}\left(A^{(2)}\right) \varepsilon_{1}\left(A^{(2)}\right)=1$, and it is not necessary to perform row exchanges.

In the rest of cases $(t>3)$, we can prove, considering a similar reasoning as above, that it is not necessary reversing the ordering of the rows.

Example 4. The matrix

$$
A=\left(\begin{array}{ccccc}
0 & -2 & -12 & -24 & -16 \\
\cline { 1 - 1 } & -31 & -138 & -244 & -152 \\
-18 & -171 & -593 & -896 & -500 \\
-54 & -405 & -1128 & -1379 & -622 \\
-54 & -351 & -801 & -717 & -173
\end{array}\right)
$$

is ASTN, i.e., ASSR (type-II staircase) with signature $\varepsilon=(-1,-1,-1,-1,-1)$.

The sequence of matrices obtained by carrying out NE with two-determinant pivoting strategy is the following

$A=\widetilde{A}^{(1)} \rightarrow A^{(1)} \rightarrow \widetilde{A}^{(2)} \rightarrow A^{(2)} \rightarrow \widetilde{A}^{(3)}=A^{(3)} \rightarrow \widetilde{A}^{(4)}=A^{(4)} \rightarrow \widetilde{A}^{(5)}=A^{(5)}$.

We can observe that row exchanges are only necessary in steps $t=1,2$.

\section{Acknowledgements}

This work has been partially supported by the Spanish Research Grant MTM2012-31544 and under Grant TEC2012-38142-C04-04 from Ministry of Education and Science, Government of Spain.

\section{References}

[1] P. Alonso, J. Delgado, R. Gallego, J.M. Peña, A collection of examples where Neville elimination outperforms Gaussian elimination, Appl. Math. Comput. 216 (2010) 2525-2533. 
[2] P. Alonso, J.M. Peña And M.L. Serrano, On the characterization of almost strictly sign regular matrices, J. Comput. Appl. Math. 275 (2015) 480-488.

[3] P. Alonso, J.M. Peña and M.L. Serrano, Almost strictly totally negative matrices: an algorithmic characterization, J. Comput. Appl. Math. 275 (2015) 238-246.

[4] T. Ando, Total positive matrices, Linear Algebra Appl. 90 (1987) 165219.

[5] R. Cantó, B. Ricarte, A. Urbano, Full rank factorization in quasi$L D U$ form of totally nonpositive rectangular matrices, Linear Algebra Appl. 440 (2014) 61-82.

[6] V. Cortés, J.M. PeÑa, Sign regular matrices and Neville elimination, Linear Algebra Appl. 421 (2007) 53-62.

[7] V. Contés, J.M. PEÑA, A stable test for strict sign regularity, Mathematics of Computation 264 (2008) 2155-2171.

[8] S.M. Fallat, P. Van Den Driessche, On matrices with all minors negative, Electronic J. of Linear Algebra 7 (2000) 92-99.

[9] S.M. Fallat, Ch.R. Johnson, Totally Nonnegative Matrices, Princeton University Press, 2011.

[10] M. Gasca, J.M. PeñA, Total positivity and Neville elimination, Linear Algebra Appl. 165 (1992) 25-44.

[11] M. Gasca, C.A. Micchelli, J.M. Peña, Almost strictly totally positive matrices, Numer. Algorithms 2 (1992) 225-236.

[12] M. Gassó, J.R. Torregrosa, A totally positive factorization of rectangular matrices by the Neville elimination, SIAM J. Matrix Anal. Appl. 25 (2004) 986-994.

[13] L. Gemignani, Neville elimination for rank-structured matrices, Linear Algebra Appl. 428 (2008) 9781-991.

[14] N.J. Higham, Accuracy and Stability of Numerical Algorithms, Second Edition, SIAM, Philadelphia, 2002. 
[15] R. Huang, J. LiU, L. Zhu, Nonsingular almost strictly sign regular matrices, Linear Algebra Appl. 436 (2012) 4179-4192.

[16] J.M. PEÑA, On nonsingular sign regular matrices, Linear Algebra Appl. 359 (2003) 91-100.

[17] A. Pinkus, Totally Positive Matrices, Cambridge University Press, Cambridge, 2010. 